\title{
Chapter 7 \\ Novel Approaches in Surgical \\ Management: How to Assess Surgical \\ Margins
}

\section{Frail Biological Basis with Promising Future Perspectives}

\author{
Marco Ferrari, Nausica Montalto, and Piero Nicolai
}

\section{Introduction}

Understanding the physical interface between tumor and host is a fascinating topic, as it dictates our current ability to appreciate the mechanisms of local growth of tumor and plan a resection with an adequate cuff of surrounding normal tissues. Despite many uncertainties regarding the definition of "adequate margins" that should be achieved by surgeons, there is strong evidence that clear resection margins are one of the main predictors of local control and overall survival in carcinomas of the upper aerodigestive tract. As a consequence, the presence of positive margins together with extranodal extension are the main factors supporting the use of chemotherapy in association with radiotherapy in the adjuvant setting $[1,2]$.

The present manuscript provides a basic historical, biological, and practical background on the concept of margins, which is essential to appreciate the importance of future perspectives in the field of margin control for tumors of the head and neck. 


\section{Historical Background: The Concept of "Margin"}

The concept of margin in oncologic surgery is almost six centuries younger than the word "cancer", which was coined by Hippocrates in view of the appearance of blood vessels surrounding a tumor and resembling the claws of a crab [3, 4]. Thereafter, cancer was considered mostly as a "humoral disease", which was consequently deemed as non-curable through simple surgical excision. Galen should be credited for being the first to hypothesize that cancer can infiltrate surrounding tissue even beyond the sensitivity of the naked eye, an intuition driven by the observation that tumors tend to regrow in scars [5]. This assumption led to conclude that cancer should be removed together with a cuff of apparently normal tissue, which still remains the pillar of surgical oncology. Although the contribute of Galen in understanding cancer is considered as controversial [6], the observation that a tumor can early return in areas adjacent to where it was completely excised can be considered as the first insight into the concept of margins. Thus, it can be estimated that the concept of "surgical margins" was born in the second century, which means almost 600 years after Hippocrates. In the nineteenth century, Virchow and Lebert observed that a cancer is formed by "cancer cells", which have the ability to invade neighboring tissues in small groups, yet not producing macroscopic changes in the early phases [5]. This new understanding of cancer provided an essential explanation to the observation of Galen, thus corroborating that cancer can be theoretically cured through excision of adjacent tissues. Despite its ancient birth, the concept of surgical margins first settled in oncologic surgery at the end of the nineteenth century, with Halsted being one of the most distinguished oncologic surgeons to concretely apply this thought to surgical practice [7]. Although biological comprehension of cancer has seen a large number of steps forward since then, the basic concept of surgical margins has remained unchanged, namely removing enough tissue to ensure that all cancer cells are included in the surgical specimen. On the other hand, the contemporary understanding of cancer biology suggests that cancer cells dissemination occurs from even early-stage tumors (also at a systemic level), thus rising some doubts on the belief that "removing all cancer cells" is the actual mechanism through which cancer is cured [8].

\section{Current Biological Rationale of Margins in Head and Neck Surgery}

The recommendation to leave a margin of normal tissue surrounding the visible tumor stands in the awareness that tumor cells can subtly extend far beyond the macroscopic boundary of the tumor. In the head and neck, oral squamous cell carcinoma represents the most frequently analyzed cancer to assess the pattern of growth towards adjacent tissues. The histologic morphology of the interface between tumor and surrounding soft tissues has been classified in five patterns with increasing 

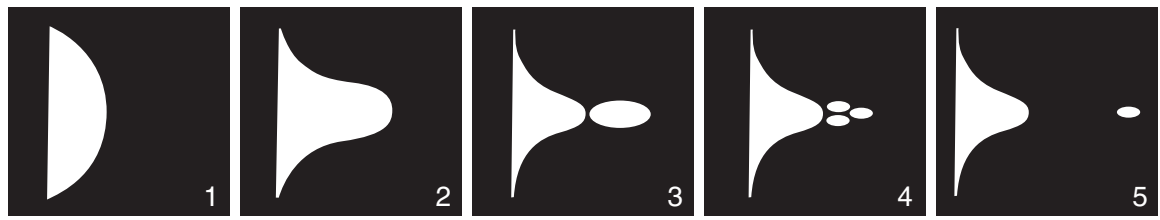

Fig. 7.1 Patterns of local invasion of soft tissues according to Brandwein-Gensler et al. [9]

degree of aggressiveness (Fig. 7.1) [9]. Type 1 pattern is defined as "broad pushing front", meaning that the tumor grows expansively and does not release groups of cells beyond its surface. Type 2 is described as "finger-like" as the tumor front displays some appendices irregularly protruding towards neighboring tissues. From type 3 to type 5, non-contiguous groups of cells with heterogeneous shape and distance from the tumor front are observed. In the type 3 front, only tumor islands, which look like "fingers" that grow up to the point of detaching from the tumor, are observed. Smaller cell groups, strands, or even single cells located within $1 \mathrm{~mm}$ from the main tumor surface fall under the definition of type 4 front. Type 5 front of invasion, finally, displays the so-called "satellites", which consist of either a cell or a group of cells located $1 \mathrm{~mm}$ beyond the tumor front. The ability to subclinically infiltrate surrounding soft tissues such as fat, striated muscles, fascial structures, and loose connective areas intuitively increases with the type of invasion front. Oral cancer was also used to analyze the pattern of invasion of bone, with special reference to the mandible. Two modalities of extension towards bone have been observed: in the erosive pattern, the tumor causes bone resorption by activating osteoclasts along a broad front of invasion; in the infiltrative pattern, tumor cells grow between bony trabecula by partially maintaining the microscopic and macroscopic bony architecture [10]. Some authors surmised that the infiltrative pattern might represent a later phase of invasion of bone compared to the erosive pattern. Parallel to these mechanisms of infiltration of adjacent tissues, cancers can acquire the ability to grow along nerves and/or vessels, which all together provides tumor cells with a dense network of pathways to move distantly from the clinically appreciable mass [11-13].

\section{Special Elements of Challenge in the Head and Neck Area}

The head and neck probably represents one of the most challenging areas of the human body to achieve adequately and homogeneously wide margins.

Although a number of factors contribute to the challenge, the need to preserve several vital functions most commonly compete with the delineation of a wide margin all along the tumor surface (Fig. 7.2a). In fact, the head and neck are dense in neurovascular structures and essential effector organs such as the brain, eyes, tongue, and larynx, which constantly place the surgeon and multidisciplinary team in front of dilemmas on resectability versus non-resectability or preservation versus ablation. 

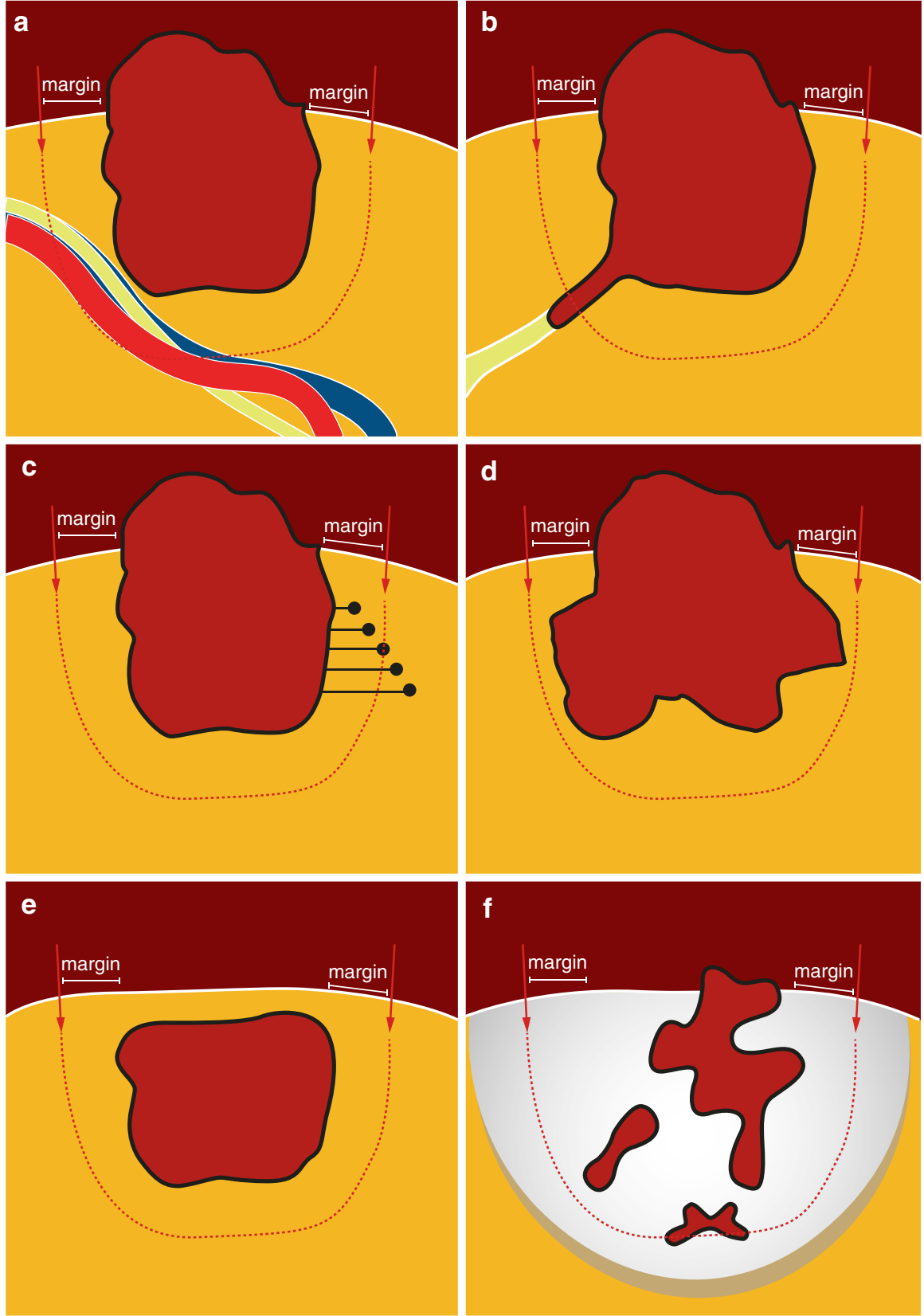

Fig. 7.2 Special elements of challenge in the management of margins in cancers of the head and neck. (a) Adjacency to critical neurovascular structures. (b) High density of nerves and vessels providing cancer with a network of escape routes. (c) Heterogeneous propensity towards subclinical extension into adjacent tissues. (d) Complex 3-dimensional shape. (e) Deep location of the tumor. (f) Multifocal tumor dispersed into previously treated tissues 
The density of neural and vascular structures also provides cancers with a dense network of potential escape routes (Fig. 7.2b). This further complicates the management of tumors displaying perineural and lymphovascular spread, as the vectors of microscopic growth of the disease might be numerous, thus making the genuine extension of the tumor deeply counterintuitive compared to the macroscopic shape of the lesion.

Biological heterogeneity is another element of complexity characterizing tumors of the head and neck. Besides the well-known variety of cancer types that exquisitely affect specific areas (i.e., sinonasal tract, salivary glands), several degrees of biological aggressiveness have been observed within a single histology (Fig. 7.2c). There is evidence that tumors pertaining to the same histological category can display widely different propensity to grow beyond the macroscopic boundaries of the lesion through budding, satellitosis, pagetoid growth, perineural spread, permeative bone invasion, or other mechanisms [14-21]. This fact poses an additional challenge, since a tumor, even though labelled with a reliable preoperative diagnosis, might potentially be amenable to a "close-margin" excision (i.e., when microscopic local extension is limited) or could instead require a "wide-margin" resection (i.e., when microscopic groups of cells deeply invade adjacent tissues) as far as is known prior to surgery.

The 3-dimensional shape of the tumor also hinders adequate and regular delineation of margins (Fig. 7.2d). While often resembling a plaque or a sphere in the early phases of growth, advanced tumors of the head and neck acquire a 3-dimensional morphology that mirrors the complexity of subsite anatomy. This translates into a substantially increased chance of misorienting the plane of dissection with respect to one or other components of the tumor [22].

The deep location of a tumor, which means that the lesion is located underneath an uninvolved epithelial plane, is not a rarity in the head and neck (Fig. 7.2e). It can result from either the origin of the tumor (e.g., salivary cancers, mesenchymal tumors), its growth pattern (i.e., submucosal growth in mucosal carcinomas) or tumor history (e.g., deep or nodal recurrences). Cancers with no superficial components force surgeons to infer the 3-dimensional configuration of the lesion based on imaging, palpation, and knowledge of anatomy, yet with a non-negligible risk for the resection to be misled.

Finally, improvement and implementation of non-surgical strategies bring to the operating theater an increasing number of patients with a tumor recurring within an irradiated and/or medically treated area (Fig. 7.2f). Similarly, refinements in surveillance strategies allow identification of post-surgical recurrences that are often suitable for surgical re-excision. Post-treatment presentation frequently implies a cancer that is multifocally dispersed within uninvolved yet deeply altered tissues, thus remarkably increasing the chance of leaving microscopic residual disease irrespective of the attention posed towards margin delineation.

These elements being considered altogether, surgical margins have been unsurprisingly a hot topic in head and neck oncology over the last decades. 


\section{Practical Determinants of Margin}

Owing to the aforesaid elements of complexity, oncologic surgeons have developed strategies to optimize margin delineation. Similar to the principles guiding elective treatment of lymph node levels, these strategies are probabilistic in nature, meaning that they are intended to maximize the probability to also include the occult portion of the disease in the resection. This, however, has the cost to unnecessarily resect uninvolved tissue in some patients, or to remove an insufficient thickness of microscopically involved tissue in others.

Three main theoretical approaches have supported the establishment of surgical rules to properly delineate margins.

The "metric approach" consists of the identification of a spatial cut-off that ensures all tumor cells are included in the resection in the majority of cases [23]. This can be objectively measured at definitive pathology. Since the distance between tumor and specimen surface shrinks during intraoperative cutting and throughout post-surgical processing, the actual margin thickness needs to be estimated. In oral cancer, for instance, since a $5 \mathrm{~mm}$ pathologic margin was identified as a prognostic cut-off in several studies, a shrinkage rate of the surgical specimen accounting for $21-32 \%$ and varying with tissue type and size, at least a $1-\mathrm{cm}$ actual margin is precautionarily recommended [24, 25]. Main argumentations against the metric approach are that a universal cut-off can be adequate, excessive, or insufficient depending upon histology and tumor-specific biology, and that $1 \mathrm{~cm}$ margin is hardly ever achievable in some head and neck sites (i.e., sinonasal tract, skull base).

The "barrier approach" is based on the assumption that tumor expansion is contained by some anatomical structures, which usually consist of fascial layers, muscles, or bones [23]. This approach leads surgeons to identify and follow specific anatomical planes that surround the tumor, even though it implies to delineate the dissection plane with an irregular distance from the tumor surface. The main flaws of this approach are in the poor recognizability of some of these barrier-structures at definitive pathology, alongside the scarce demonstrability that they actually serve as barriers against tumor local progression.

The "compartment approach", finally, is based on the surmise that tumor cells tend to follow specific anatomical structures or vectors dictated by tissue architecture [26]. Though sounding similar to the barrier approach, this way of conceiving tumor progression is less optimistic on the capability of some structures to prevent local cancer progression. Rather, cancer cells would expand owing to a "pressure growth" that pushes cancers towards the pathways of least resistance (e.g., between muscular fibers or fascicles).

As for all competing theoretical models aimed at explaining a biological phenomenon, the reality probably lies somewhere in the middle. Most likely, cancers progress through preferential pathways (either because of least resistance or due to a biological gain of function such as perineural spread), while also stochastically infiltrating surrounding tissues with some structures (e.g., bone, cartilage) probably 
serving as physical barriers against tumor expansion. Moreover, distribution between these modalities of local expansion can obviously vary among malignancies.

A paradoxical fact on recommendations for margin width lies in the techniquedependent threshold defining "clear margins". A cancer of the upper aerodigestive tract would be defined as completely resected with a threshold of $5 \mathrm{~mm}$ of pathologically uninvolved tissue if operated on with open surgery, $2-5 \mathrm{~mm}$ if through transoral robotic surgery, $0.5-2 \mathrm{~mm}$ if via transoral laser surgery, and regardless of metric measurements provided that adjacent structures are not infiltrated in case endoscopic transnasal resection has been performed [27-36]. On the one hand, this difference is understandable as it expresses the need to define as either "adequate" or "inadequate" a resection performed with a given technique. On the other, it reflects that the definition of margin is currently far from being biology-driven [37].

\section{“Frailty” of Cutting Through Healthy Tissue}

The concept of "free margin" grounds on the belief that tissue uninvolved by cancer is healthy. However, evidence dating back to the 1990s suggested that tissues surrounding mucosal cancers bear molecular alterations typically found in malignancies [38]. These observations are in agreement with the multistep model that explains cancer development and progression. In fact, precancerous cells that gradually accumulate all the mutations necessary to become cancer also proliferate, thus giving rise to a number of cells that are preconditioned towards malignant transformation. This might also explain the propensity of cancers induced by long-term exposure to a risk factor (i.e., tobacco smoking) towards recurrence, field cancerization, and synchronous/metachronous malignancies. Consequently, instead of conceiving cancer as a well-defined mass, preconditioning of the surrounding mucosa contributes to make it more comparable to an ill-defined "cloud" of genetic alterations centered around the visible disease and variably extending to the adjacent mucosa (Fig. 7.3).

\section{Current Intraoperative Margin Evaluation}

For mucosal cancers, which represent the majority of head and neck malignant tumors, delineation of margins is required on both the superficial aspect, meaning that the surgeon has to decide how far from the visible tumor the mucosa has to be cut, and during dissection of deep tissues. For superficial delineation of margins, surgeons rely on sight and palpation, with some technologies (e.g., narrow band imaging) augmenting the ability to identify altered tissues mostly owing to optical changes $[39,40]$. Delineation of deep margins is based on palpation, imaging 
Fig. 7.3 Discrepancy between the common representation of cancer (a) and actual distribution of precancerous alterations in adjacent tissues $(\mathbf{b})$
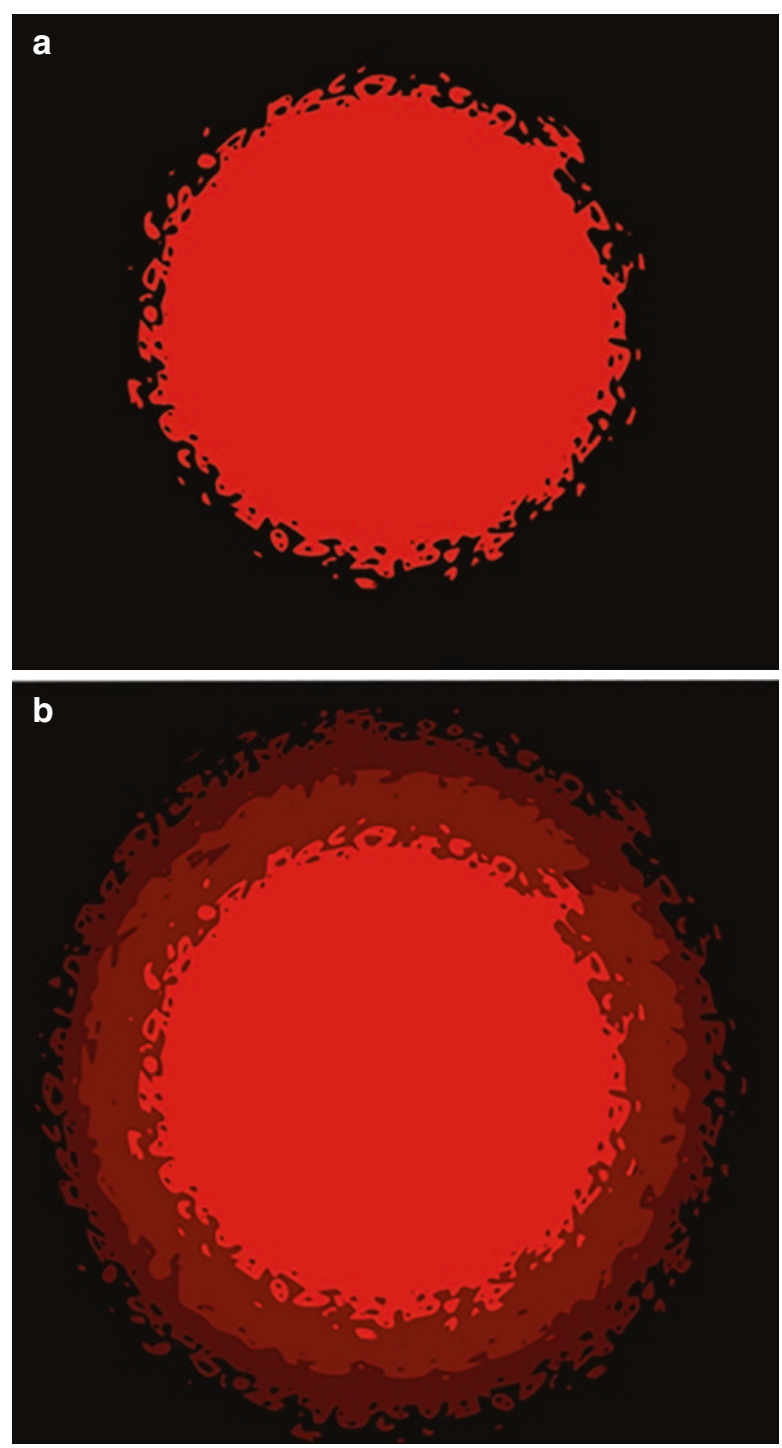

interpretation, and the consequent 3-dimensional configuration that the surgeon creates in his/her mind. Sight is currently excluded from the ideal strategies to define the deep margin of resection, as it would imply the deep portion of the tumor to be exposed, which is a suboptimal scenario as opposed to leaving the tumor surrounded by a cuff of normal tissue.

Frozen sections allow intraoperative microscopic assessment of resection margins. Two main approaches to perform frozen sections for margin assessment are traditionally available: the defect-driven (also defined as patient-driven) technique consists of sampling the surgical bed, whereas in the specimen-driven technique 
tissues to be analyzed are harvested from the surgical specimen. There is no consensus on which technique yields the best accuracy in terms of intraoperative margin evaluation. Some evidence suggests that specimen-driven frozen sections might provide a higher chance of achieving wide negative margins as compared to defectdriven approach [41]. Moreover, positive frozen sections on the surgical specimen may also represent an independent negative prognostic factor, whereas defectdriven frozen sections have not been demonstrated to carry any relevant prognostic information [42]. This could be explained by the fact that sampling on the surgical specimen leads the surgeon to address the most critical margin relative to the palpable mass, whereas analysis of the surgical bed requires inferring the initial situation of the tumor. However, some authors have reported that circumferential sampling of the surgical bed has an almost excellent negative predictive value, though with suboptimal positive predictive value [43]. Irrespective of the specific technique employed to sample tissue to be sent for frozen section analysis, a metaanalysis demonstrated that achieving negative margins by extending the resection based on a positive frozen section does not equate to an initially negative margin, nor does it significantly increase the local control rate [44]. These data should not be misinterpreted as suggesting uselessness of achieving negative margins through additional resection following a positive frozen section. In fact, in the same metaanalysis, local recurrence-free survival of patients with positive margins is reported being close-to-significantly $(\mathrm{p}=0.055)$ worse compared to those with negative margins achieved through additional specimens on a positive frozen section [44]. As a consequence, one can conclude that obtaining negative margins upfront represents the best case scenario from a prognostic standpoint, but radicalization on a positive frozen section is still to be recommended based on the currently available data.

\section{Future Directions: "Know Your Enemy"}

Borrowing the aphorism of Sun Tzu from "The art of war", the first step to improve our ability to locally control cancer should consist of "knowing cancer". In particular, it is a common observation that every head and neck cancer has its own specificity in terms of local progression, which is not reliably expressed by the current systems of classifying and describing tumors.

For instance, it has been demonstrated that tongue squamous cell carcinoma has a particular propensity to subclinically invade the so-called "T-N tract", which roughly corresponds to the connective space including the sublingual area up to the level IB [45]. This confirms that tongue cancer can grow eccentrically with respect to the epicenter of clinically appreciable disease, which has not been observed in other oral cavity subsites whose cancerization shares analogous epidemiological and histopathological characteristics. This data being acquired, a modification of the surgical technique defined as "compartmental tongue surgery" has been implemented by some groups, aiming at addressing this particular characteristic of tongue cancer. Indeed, based on preliminary and retrospective data, compartmental tongue 
resection seems to provide improved oncologic outcomes compared to standard wide-margin resection $[26,46]$. These findings possibly confirm that focusing attention on the most probable escaping route of tumor might translate into better control of cancer.

Another example of deepening the understanding of cancer local behavior is the relationship between histologic growth pattern and topographic gross extension. For instance, it has been revealed that perineural and lymphovascular invasion substantially drive local extension of cancers of the maxillary sinus regardless of their histology [47]. In particular, tumors displaying lymphovascular invasion tend to grow with a caudal direction and give nodal metastases, while those with perineural invasion more frequently invade superior, medial, and posterior structures. Should detection of perineural and lymphovascular invasion be reliably detectable before surgery, the resection could be extended accordingly towards the most critical areas.

In view of this evidence, head and neck oncologic surgeons should be avid in knowing the local behavior of cancers with a histology-, site-, and possibly biologylevel precision. Therefore, future research on local tumor extension in the head and neck should primarily assess the relationship between the cancer's specificities and escape routes, in order to guide surgeons towards the most critical areas and possibly improve outcomes.

\section{Future Directions: Enhanced Tumor Visualization}

Another strategy to improve local control is to augment the way cancer is "seen" during ablation. The most promising and accessible technology to support this refinement is represented by surgical navigation systems. Although most frequently employed to minimize intraoperative complications and optimize precision of reconstruction, cross-sectional imaging-based navigation could also provide the surgical team with a more precise image of tumor extension. This has been shown in a preclinical setting, where the employment of navigation with 3-dimensional rendering of the tumor extension significantly increased the adequacy of margin delineation in models of advanced cancers variably extending within the craniomaxillofacial skeleton [22]. Over a total of 381 simulated osteotomies, the use of surgical navigation decreased the rate of gross margin involvement from $18.1 \%$ to $0.0 \%$. Moreover, some groups have published their experience in using navigation to improve the margin status of resections of advanced cancers of the head and neck, showing encouraging results [48-50]. Despite the limited number of patients reported in these preliminary experiences ( 24 overall), the employment of navigation led to obtain free margins in a high percentage of patients affected by locally advanced cancer of the head and neck.

By basing the 3-dimensional representation of the tumor on radiologic data, navigation-guided resections might also benefit from incorporating relevant information into cancer rendering. For instance, the tumor can be rendered together with an isotropic expansion to provide a visual representation of a metric margin. 
Moreover, cancer rendering could also include fusion of functional and cross sectional imaging, possibly increasing the accuracy of tumor mapping [50]. In this sense, whichever future methodology is capable of better depicting the actual tumor extension could be incorporated in the representation of tissue to be resected through surgical navigation.

However, the accuracy of surgical navigation is constrained by precise and lasting registration alongside the presence of a bony framework that limits motions of soft tissue. For this reason, navigation is most likely useful in the setting of tumors strictly attached to the craniomaxillofacial skeleton, whereas cancers invading mostly soft tissues would be less accurately rendered.

\section{Future Directions: Augmented Mapping of the Surgical Bed}

The latest and most promising advent in the field of surgical margins control is application of bio-optical imaging technologies to search for tumor localizations that would otherwise be undetectable by the naked eye [51]. Employment of this technology to improve delineation of the superficial margin of resection has been already demonstrated to be beneficial. On the contrary, optical imaging to detect potential residues of the tumor into the surgical bed and accordingly guide frozen section is still an everchanging field. The most promising optical imaging modalities which could meet this need are fluorescence-based imaging, hyperspectral imaging, and Raman spectroscopy. Fluorescence-based imaging relies on either natural (i.e., autofluorescence imaging) or targeted fluorescence (i.e., through biological probes attached to fluorophores) of cancer tissue. Hyperspectral imaging consists of dividing electromagnetic waves beyond the 3-band division of the human eye and even beyond the spectrum of visible light. By collecting and elaborating this optical information, it is possible to infer biological information of a tissue under analysis. Raman spectroscopy is able to depict the molecular fingerprint of a tissue by taking advantage of light scattering as a consequence of vibration of intramolecular bonds. All these imaging modalities rely on the common principle of collecting bio-optical characteristics of tissues and render them in a way that is appreciable to the surgeon's eye.

Recently, van Keulen et al. published a series of 20 patients who were operated on for head and neck squamous cell carcinoma by targeted fluorescence-surgery [52]. All patients were injected with panitumumab, an anti-epidermal growth factor receptor (EGFR) monoclonal antibody, conjugated to the fluorophore IRDye800CW. The surgeon could therefore visualize in real time the distribution of EGFR through a handheld camera prior to incise tissues. The authors demonstrated that tumor-to-background ratio, which represents the ability to distinguish the tumor from surrounding tissues, was satisfactory irrespective of age, gender, tumor size and site, and EGFR expression. Though preliminary in nature, these data are encouraging, as they demonstrate feasibility of the workflow and suggest that targeted-fluorescence imaging is reliable. Analysis of the actual benefit of this technology in terms of intraoperative margin status evaluation will represent an essential future step. 
Halicek et al. published a study on 293 fresh specimens obtained from resection of head and neck squamous cell carcinomas in 102 patients and analyzed with reflectance-based hyperspectral imaging [53]. The authors found that hyperspectral imaging could distinguish squamous cell carcinoma from uninvolved tissue with an area-under-curve ranging between 0.80 and 0.90 compared to histopathological microscopic evaluation. The time span required to obtain hyperspectral-based evaluation of the surgical specimen was estimated to be around $2 \mathrm{~min}$. This study provided promising data on the classification performance of hyperspectral imaging calculated from a large dataset. However, application of this methodology to the surgical bed would require optimization for potential confounders such as blood and cauterized tissues.

Barroso et al. have demonstrated the utility of Raman spectroscopy in identifying positive margins on 26 mandibulectomy specimens, with diagnostic accuracy as high as $95 \%$ [54]. Yu et al. achieved a $99.3 \%$ sensitivity and $94.3 \%$ specificity in distinguishing tongue squamous cell carcinoma with respect to normal tissue by applying a deep learning method to Raman spectral data obtained from 24 fresh specimens [55].

The above-mentioned references represent just selected publications among a large and constantly increasing number of studies demonstrating and progressively refining the diagnostic performance of bio-optical imaging techniques on fresh tissues harboring cancer. The following step will probably be to apply these technologies intraoperatively and quantify the actual benefit they can confer to outcomes.

\section{Conclusions}

Adequate control of margins is an urgent need in head and neck surgical oncology. Our current understanding of local progression of cancer is still inadequate, especially considering the variety of histologies and biological behaviors characterizing the head and neck area. Consensus should be reached to obtain a solid and biologydriven definition of "adequate margins", which could be transversally applied to a given cancer irrespective of the surgical technique employed to excise it. On the other hand, technologies such as surgical navigation and bio-optical imaging will probably be implementing our current way of ablating cancers, possibly translating into better delineated surgical specimens and improved outcomes.

\section{References}

1. Bernier J, Domenge C, Ozsahin M, et al. Postoperative irradiation with or without concomitant chemotherapy for locally advanced head and neck cancer. NEngl J Med. 2004;350(19):1945-52. https://doi.org/10.1056/NEJMoa032641.

2. Cooper JS, Pajak TF, Forastiere AA, et al. Postoperative concurrent radiotherapy and chemotherapy for high-risk squamous-cell carcinoma of the head and neck. N Engl J Med. 2004;350(19):1937-1944+2019. https://doi.org/10.1056/NEJMoa032646. 
3. Deeley TJ. A brief history of cancer. Clin Radiol. 1983;34(6):597-608. https://doi.org/10.1016/ S0009-9260(83)80405-X.

4. di Lonardo A, Nasi S, Pulciani S. Cancer: we should not forget the past. J Cancer. 2015;6(1):29-39. https://doi.org/10.7150/jca.10336.

5. Fonseca R. Oral and maxillofacial. Surgery. 2017; https://doi.org/10.1016/ B978-0-323-26278-1.00011-8.

6. Faguet GB. A brief history of cancer: age-old milestones underlying our current knowledge database. Int J Cancer. 2015;136(9):2022-36. https://doi.org/10.1002/ijc.29134.

7. Halsted WS. The results of operations for the cure of cancer of the breast performed at the Johns Hopkins Hospital from June, 1889, to January, 1894. Ann Surg. 1894;20(5):497-555. https://doi.org/10.1097/00000658-189407000-00075.

8. Wolf GT. Surgical margins in the genomic era: the Hayes Martin lecture, 2012. Arch Otolaryngol - Head Neck Surg. 2012;138(11):1001-13. https://doi.org/10.1001/2013. jamaoto.82.

9. Brandwein-Gensler M, Teixeira MS, Lewis CM, et al. Oral squamous cell carcinoma: histologic risk assessment, but not margin status, is strongly predictive of local disease-free and overall survival. Am J Surg Pathol. 2005;29(2):167-78. https://doi.org/10.1097/01. pas.0000149687.90710.21.

10. Shaw RJ, Brown JS, Woolgar JA, Lowe D, Rogers SN, Vaughan ED. The influence of the pattern of mandibular invasion on recurrence and survival in oral squamous cell carcinoma. Head Neck. 2004;26(10):861-9. https://doi.org/10.1002/hed.20036.

11. Aleskandarany MA, Sonbul SN, Mukherjee A, Rakha EA. Molecular mechanisms underlying lymphovascular invasion in invasive breast cancer. Pathobiology. 2015;82(3-4):113-23. https://doi.org/10.1159/000433583.

12. Marchesi F, Piemonti L, Mantovani A, Allavena P. Molecular mechanisms of perineural invasion, a forgotten pathway of dissemination and metastasis. Cytokine Growth Factor Rev. 2010;21(1):77-82. https://doi.org/10.1016/j.cytogfr.2009.11.001.

13. Amit M, Na'Ara S, Gil Z. Mechanisms of cancer dissemination along nerves. Nat Rev Cancer. 2016;16(6):399-408. https://doi.org/10.1038/nrc.2016.38.

14. Maffeis V, Cappellesso R, Galuppini F, et al. Tumor budding is an adverse prognostic marker in intestinal-type sinonasal adenocarcinoma and seems to be unrelated to epithelial-mesenchymal transition. Virchows Arch. 2020;477:241-8. https://doi.org/10.1007/s00428-020-02748-1.

15. Garden AS, Weber RS, Morrison WH, Ang KK, Peters LJ. The influence of positive margins and nerve invasion in adenoid cystic carcinoma of the head and neck treated with surgery and radiation. Int J Radiat Oncol Biol Phys. 1995;32(3):619-26. https://doi. org/10.1016/0360-3016(95)00122-F.

16. Liu S-A, Wang C-C, Jiang R-S, Lee F-Y, Lin W-J, Lin J-C. Pathological features and their prognostic impacts on oral cavity cancer patients among different subsites - a singe institute's experience in Taiwan. Sci Rep. 2017;7(1):7451. https://doi.org/10.1038/s41598-017-08022-w.

17. Martins-Andrade B, Dos Santos Costa SF, Sant'ana MSP, et al. Prognostic importance of the lymphovascular invasion in head and neck adenoid cystic carcinoma: a systematic review and meta-analysis. Oral Oncol. 2019;93:52-8. https://doi.org/10.1016/j.oraloncology.2019.04.014.

18. Adel M, Kao H-K, Hsu C-L, et al. Evaluation of lymphatic and vascular invasion in relation to clinicopathological factors and treatment outcome in oral cavity squamous cell carcinoma. Medicine (Baltimore). 2015;94(43):e1510. https://doi.org/10.1097/MD.0000000000001510.

19. Ho YY, Wu TY, Cheng HC, Yang CC, Wu CH. The significance of tumor budding in oral cancer survival and its relevance to the eighth edition of the American Joint Committee on Cancer staging system. Head Neck. 2019;41(9):2991-3001. https://doi.org/10.1002/hed.25780.

20. King R, Page RN, Googe PB, Mihm MC. Lentiginous melanoma: a histologic pattern of melanoma to be distinguished from lentiginous nevus. Mod Pathol. 2005;18(10):1397-401. https:// doi.org/10.1038/modpathol.3800454.

21. Takahashi Y, Takahashi E, Nakakura S, Kitaguchi Y, Mupas-Uy J, Kakizaki H. Risk factors for local recurrence or metastasis of eyelid sebaceous gland carcinoma after wide excision with paraffin section control. Am J Ophthalmol. 2016;171:67-74. https://doi.org/10.1016/j. ajo.2016.08.028. 
22. Ferrari M, Daly MJ, Douglas CM, et al. Navigation-guided osteotomies improve margin delineation in tumors involving the sinonasal area: a preclinical study. Oral Oncol. 2019;99:104463. https://doi.org/10.1016/j.oraloncology.2019.104463.

23. Upile T, Fisher $\mathrm{C}$, Jerjes $\mathrm{W}$, et al. The uncertainty of the surgical margin in the treatment of head and neck cancer. Oral Oncol. 2007;43(4):321-6. https://doi.org/10.1016/j. oraloncology.2006.08.002.

24. Pangare TB, Waknis PP, Bawane SS, Patil MN, Wadhera S, Patowary PB. Effect of formalin fixation on surgical margins in patients with oral squamous cell carcinoma. J Oral Maxillofac Surg. 2017;75(6):1293-8. https://doi.org/10.1016/j.joms.2016.11.024.

25. Johnson RE, Sigman JD, Funk GF, Robinson RA, Hoffman HT. Quantification of surgical margin shrinkage in the oral cavity. Head Neck. 1997;19(4):281-6. https://doi.org/10.1002/ (sici)1097-0347(199707)19:4<281::aid-hed6>3.3.co;2-4.

26. Calabrese L, Bruschini R, Giugliano G, et al. Compartmental tongue surgery: long term oncologic results in the treatment of tongue cancer. Oral Oncol. 2011;47(3):174-9. https://doi. org/10.1016/j.oraloncology.2010.12.006.

27. Nichols AC, Theurer J, Prisman E, et al. Radiotherapy versus transoral robotic surgery and neck dissection for oropharyngeal squamous cell carcinoma (ORATOR): an open-label, phase 2, randomised trial. Lancet Oncol. 2019;20(10):1349-59. https://doi.org/10.1016/ S1470-2045(19)30410-3.

28. Benazzo M, Canzi P, Mauramati S, et al. Transoral robot-assisted surgery in supraglottic and oropharyngeal squamous cell carcinoma: laser versus monopolar electrocautery. J Clin Med. 2019;8(12):2166. https://doi.org/10.3390/jcm8122166.

29. Persky MJ, Albergotti WG, Rath TJ, et al. Positive margins by oropharyngeal subsite in transoral robotic surgery for T1/T2 squamous cell carcinoma. Otolaryngol - Head Neck Surg (United States). 2018;158(4):660-6. https://doi.org/10.1177/0194599817742852.

30. Cannon RB, Houlton JJ, Patel S, et al. Patterns of cervical node positivity, regional failure rates, and fistula rates for HPV+ oropharyngeal squamous cell carcinoma treated with transoral robotic surgery (TORS). Oral Oncol. 2018;86:296-300. https://doi.org/10.1016/j. oraloncology.2018.10.001.

31. Cracchiolo JR, Roman BR, Kutler DI, Kuhel WI, Cohen MA. Adoption of transoral robotic surgery compared with other surgical modalities for treatment of oropharyngeal squamous cell carcinoma. J Surg Oncol. 2016;114(4):405-11. https://doi.org/10.1002/jso.24353.

32. Vaish R, Shah S, Chaukar D. Prognostic significance of surgical margins after transoral laser microsurgery for early-stage glottic cancer. Oral Oncol. 2020;100:104511. https://doi. org/10.1016/j.oraloncology.2019.104511.

33. Peretti G, Piazza C, Cocco D, et al. Transoral CO2 laser treatment for tis-T3 glottic cancer: the University of Brescia experience on 595 patients. Head Neck. 2010;32(8):977-83. https://doi. org/10.1002/hed.21278.

34. Abdelmeguid AS, Raza SM, Su SY, et al. Endoscopic resection of sinonasal malignancies. Head Neck. 2020;42(4):645-52. https://doi.org/10.1002/hed.26047.

35. Castelnuovo P, Battaglia P, Turri-Zanoni M, et al. Endoscopic endonasal surgery for malignancies of the anterior cranial base. World Neurosurg. 2014;82(6):S22-31. https://doi. org/10.1016/j.wneu.2014.07.021.

36. Nicolai P, Castelnuovo P, Villaret AB. Endoscopic resection of sinonasal malignancies. Curr Oncol Rep. 2011;13(2):138-44. https://doi.org/10.1007/s11912-011-0151-6.

37. Meier JD, Oliver DA, Varvares MA. Surgical margin determination in head and neck oncology: current clinical practice. The results of an international American head and neck society member survey. Head Neck. 2005;27(11):952-8. https://doi.org/10.1002/hed.20269.

38. Ball VA, Righi PD, Tejada E, Radpour S, Pavelic ZP, Gluckman JL. p53 immunostaining of surgical margins as a predictor of local recurrence in squamous cell carcinoma of the oral cavity and oropharynx. Ear Nose Throat J. 1997;76(11):818-23. https://doi. org/10.1177/014556139707601109.

39. Piazza C, Del Bon F, Peretti G, Nicolai P. Narrow band imaging in endoscopic evaluation of the larynx. Curr Opin Otolaryngol Head Neck Surg. 2012;20(6):472-6. https://doi.org/10.1097/ MOO.0b013e32835908ac. 
40. Piazza C, Del Bon F, Paderno A, et al. The diagnostic value of narrow band imaging in different oral and oropharyngeal subsites. Eur Arch Oto-Rhino-Laryngol. 2016;273(10):3347-53. https://doi.org/10.1007/s00405-016-3925-5.

41. Amit M, Na'Ara S, Leider-Trejo L, et al. Improving the rate of negative margins after surgery for oral cavity squamous cell carcinoma: a prospective randomized controlled study. Head Neck. 2016;38:E1803-9. https://doi.org/10.1002/hed.24320.

42. Buchakjian MR, Ginader T, Tasche KK, Pagedar NA, Smith BJ, Sperry SM. Independent predictors of prognosis based on oral cavity squamous cell carcinoma surgical margins. Otolaryngol - Head Neck Surg (United States). 2018;159(4):675-82. https://doi. org/10.1177/0194599818773070.

43. Tirelli G, Boscolo Nata F, Gatto A, et al. Intraoperative margin control in transoral approach for oral and oropharyngeal cancer. Laryngoscope. 2019;129(8):1810-5. https://doi.org/10.1002/ lary. 27567.

44. Bulbul MG, Tarabichi O, Sethi RK, Parikh AS, Varvares MA. Does clearance of positive margins improve local control in oral cavity cancer? A meta-analysis. Otolaryngol - Head Neck Surg (United States). 2019;161(2):235-44. https://doi.org/10.1177/0194599819839006.

45. Tagliabue M, Gandini S, Maffini F, et al. The role of the T-N tract in advanced stage tongue cancer. Head Neck. 2019;41(8):2756-67. https://doi.org/10.1002/hed.25761.

46. Piazza C, Grammatica A, Montalto N, Paderno A, Del Bon F, Nicolai P. Compartmental surgery for oral tongue and floor of the mouth cancer: oncologic outcomes. Head Neck. 2019;41(1):110-5. https://doi.org/10.1002/hed.25480.

47. Ferrari M, Ioppi A, Schreiber A, et al. Malignant tumors of the maxillary sinus: prognostic impact of neurovascular invasion in a series of 138 patients. Oral Oncol. 2020;106:104672. https://doi.org/10.1016/j.oraloncology.2020.104672.

48. Catanzaro S, Copelli C, Manfuso A, et al. Intraoperative navigation in complex head and neck resections: indications and limits. Int J Comput Assist Radiol Surg. 2017;12(5):881-7. https:// doi.org/10.1007/s11548-016-1486-0.

49. Tarsitano A, Ricotta F, Baldino G, et al. Navigation-guided resection of maxillary tumours: the accuracy of computer-assisted surgery in terms of control of resection margins - a feasibility study. J Cranio-Maxillofacial Surg. 2017;45(12):2109-14. https://doi.org/10.1016/j. jcms.2017.09.023.

50. Feichtinger M, Pau M, Zemann W, Aigner RM, Kärcher H. Intraoperative control of resection margins in advanced head and neck cancer using a 3D-navigation system based on PET/ CT image fusion. J Cranio-Maxillofacial Surg. 2010;38(8):589-94. https://doi.org/10.1016/j. jems.2010.02.004.

51. Wu C, Gleysteen J, Teraphongphom NT, Li Y, Rosenthal E. In-vivo optical imaging in head and neck oncology: basic principles, clinical applications and future directions review. Int $\mathrm{J}$ Oral Sci. 2018;10(2). https://doi.org/10.1038/s41368-018-0011-4

52. van Keulen S, Nishio N, Fakurnejad S, et al. Intraoperative tumor assessment using real-time molecular imaging in head and neck cancer patients. J Am Coll Surg. 2019;229(6):560-567. e1. https://doi.org/10.1016/j.jamcollsurg.2019.09.007.

53. Halicek M, Dormer JD, Little JV, et al. Hyperspectral imaging of head and neck squamous cell carcinoma for cancer margin detection in surgical specimens from 102 patients using deep learning. Cancers (Basel). 2019;11(9). https://doi.org/10.3390/cancers11091367

54. Barroso EM, ten Hove I, Bakker Schut TC, et al. Raman spectroscopy for assessment of bone resection margins in mandibulectomy for oral cavity squamous cell carcinoma. Eur J Cancer. 2018;92:77-87. https://doi.org/10.1016/j.ejca.2018.01.068.

55. Yu M, Yan H, Xia J, et al. Deep convolutional neural networks for tongue squamous cell carcinoma classification using Raman spectroscopy. Photodiagn Photodyn Ther. 2019;26:430-5. https://doi.org/10.1016/j.pdpdt.2019.05.008. 
Open Access This chapter is licensed under the terms of the Creative Commons Attribution 4.0 International License (http://creativecommons.org/licenses/by/4.0/), which permits use, sharing, adaptation, distribution and reproduction in any medium or format, as long as you give appropriate credit to the original author(s) and the source, provide a link to the Creative Commons license and indicate if changes were made.

The images or other third party material in this chapter are included in the chapter's Creative Commons license, unless indicated otherwise in a credit line to the material. If material is not included in the chapter's Creative Commons license and your intended use is not permitted by statutory regulation or exceeds the permitted use, you will need to obtain permission directly from the copyright holder. 